\title{
Shh promotes sweat gland cell maturation in three- dimensional culture
}

\author{
Zhijian Huang • Yunfang Zhen • \\ Wei Yin $\cdot$ Zhourui Ma $\cdot$ Liya Zhang
}

Received: 6 December 2015/ Accepted: 16 February 2016/Published online: 23 February 2016

(C) The Author(s) 2016. This article is published with open access at Springerlink.com

\begin{abstract}
Sweat gland (SG) cells forming SG tubule-like structures in 3D culture, this is one of the most important methods to identify the biological function of SG cells and stem cells-derived SG-like cells, but also the important way on research of SG regeneration in vitro. In this study, we seeded human fibroblasts and SG cells in gels and used immunohistochemistry to confirm whether SG tubule-like structures formed. Fibroblasts are necessary factor in the process of SG cells maturation and forming SG's
\end{abstract}

Zhijian Huang and Yunfang Zhen have contributed equally to this work.

Electronic supplementary material The online version of this article (doi:10.1007/s10561-016-9548-7) contains supplementary material, which is available to authorized users.

Z. Huang · W. Yin $\cdot$ Z. Ma

Department of Burn and Plastic Surgery, Children's

Hospital of Soochow University, Suzhou,

Jiangsu Province, People's Republic of China

\section{Y. Zhen}

The Center of Diagnosis and Treatment for Children's Bone Diseases, Children's Hospital of Soochow University, Suzhou, Jiangsu Province, People's Republic of China

\section{Zhang ( $₫)$}

Department of Neurology, Children's Hospital of

Soochow University, Suzhou, Jiangsu Province, People's

Republic of China

e-mail: liya_zhang1516@163.com secretory region in 3D culture. Further experimentation revealed that Sonic hedgehog (Shh) was secreted by fibroblasts within the 3D culture. By adding Shh protein to $3 \mathrm{D}$ culture, there had more SG tubule-like structures formed. These results suggest that Shh is an important factor during the process of forming SG tubule-like structures in 3D cultures, and adding Shh recommbinant protein could promote SG cell maturation and enhance the efficiency of structure formation.

Keywords Fibroblasts $\cdot$ Shh $\cdot$ Sweat gland tubulelike structure $\cdot 3 \mathrm{D}$ culture

\section{Introduction}

Sweat glands are one of the most important skin appendages; their main function is to perspire and control body temperature. Sweat gland development is complicated, and the production of sweat glands ceases after birth; once sweat glands are destroyed, they cannot regenerate (Saga 2002). There are millions of burn injury patients every year across the world. Of these patients, approximately $10 \%$ suffer severe full-thickness burns to their skin (Fu et al. 2006). A common treatment for burn injuries is the use of skin allografts to cover the wounds (Cuono et al. 1986; Naoum et al. 2004; Burd and Chiu 2005). These, however, not involved sweat gland, increasing the patient's pain.

The development of tissue engineering has opened up a new path for the repair of large skin lesions $(\mathrm{Fu}$ 
et al. 2005). The reconstruction of skin that possesses not only the epidermal and dermal portions but also skin appendages is important. Therefore, regenerating the structure of sweat glands is an important clinical problem. Accumulating studies have focused on the regeneration of sweat glands. Researchers have found that bone marrow mesenchymal stem cells (MSCs) can differentiate into sweat gland-like cells in vitro. Following the transplantation of these cells into nude mice or deep burn injury patients, damaged sweat glands were reconstructed (Sheng et al. 2009).

With the recent development of extracellular matrices, the 3D reconstruction of sweat glands in vitro has become possible (Kleinman and Martin 2005). Whether sweat gland cells can form tubule-like structures in 3D culture, this is an important indicator for identification of sweat gland and stem cellsderived sweat gland cells and their biological function; this is also an important method for sweat gland tissue engineering research. A 3D culture system has been established using Matrigel, and sweat gland cells cultured in this system can form tubule-like structures (Li et al. 2013). Furthermore, a collagen type I solution and Matrigel were mixed and epidermal growth factor (EGF)-loaded microspheres were added as both a slow-release depot of growth factors and a delivery vehicle for the SG cells. SG cells in this system could also form sweat gland tubule-like structures (Huang et al. 2010).

However, what factors does fibroblast secrete of this process remain unclear. In this study, we found that fibroblast was an essential factor for sweat gland cell forming tubule-like structures in 3D culture system; we demonstrated that Shh was an important factor for sweat gland cells form tubule-like structures in $3 \mathrm{D}$ culture, and was secreted by fibroblasts; we also found that adding extra Shh can enhance the efficiency of structure formation.

\section{Materials and methods}

Isolation and culture of human sweat gland cells

Infant polydactyly skin samples were obtained from the Children's Hospital affiliated to Soochow University and were donated with the parents' informed consent. The process was reviewed and approved by the Institutional Review Board and the Ethics
Committee of the First Affiliated Hospital of the PLA General Hospital (Huang et al. 2010). Tissues were washed in phosphate buffered saline (PBS) with penicillin/streptomycin (Gibco) for $10 \mathrm{~min}$ at room temperature. After removing subcutaneous fat, the skin was minced into $1 \mathrm{~mm}^{3}$ pieces and washed in PBS without penicillin or streptomycin. Then, the pieces of skin were treated with dispase $(1 \mathrm{mg} / \mathrm{ml}$; Roche) for $18 \mathrm{~h}$. After the dispase treatment, the epidermis and dermis were separated, using nippers to isolate the dermis from the tissue. The dermis was then treated with collagenase type IV $(2.5 \mathrm{mg} / \mathrm{ml}$; Sigma, USA) for $1 \mathrm{~h}$ at $37^{\circ} \mathrm{C}$. After digestion, the sweat glands were dissociated from the surrounding collagen and fat and individually removed using a transfer pettorunder an ultraviolet-sterilized phase-contrast inverted microscope $(\times 40)$.

The SG cells were cultured in a sweat gland cellmedium(SGM), consisting of: Glucose Dulbecco's modified Eagle's medium (DMEM-F12, Gibco) supplemented with $10 \%$ FBS (Hyclone), $1 \%$ penicillin/ streptomycin (Gibco), $2 \mathrm{mM}$ L-glutamine (Gibco), $10 \times$ insulin-transferring sodium selenite solution (ITS, Gibco), $2 \mathrm{nM} / \mathrm{ml}$ triiodothyronine (T3, Sigma), $0.4 \mathrm{mg} / \mathrm{ml}$ hemisuccinate hydrocortisone (Sigma) and $10 \mathrm{ng} / \mathrm{ml}$ human recombinant epidermal growth factor (EGF, R\&D).

Isolation and culture of human dermal fibroblast cells

After isolating the epidermis and dermis, the dermis was cut into small pieces and treated with collagenase type IV for $1 \mathrm{~h}$ at $37^{\circ} \mathrm{C}$. After digestion, the mixture was sifted through mesh. The cell suspension was centrifuged and resuspended in high glucose DMEM (Gibco) supplemented with $10 \%$ FBS.

For co-cultured fibroblasts and SG cells, SG cells have stronger adherent ability than fibroblasts. We used $0.25 \%$ Tyrisin/EDTA (Gibco) to digest cocultured cells for $1 \mathrm{~min}$, fibroblasts were digested in the suspension but SG cells were still adhere on the dish, then we collected fibroblasts cultured in vitro for future experiment.

RNA isolation and real-time quantitative PCR

Total RNA was isolated from cells using the RNeasy Mini extraction kit (Qiagen, Courtaboeuf, Germany) 
according to the manufacturer's protocol. A total of $1 \mu \mathrm{g}$ RNA was used for reverse transcription. For PCR analysis, cDNA was reverse transcribed using a Reverse Transcriptase M-MLV kit (TaKaRa). For real time-PCR analysis, cDNA was reverse transcribed using a PrimeScript ${ }^{\mathrm{TM}} \mathrm{RTMaster}$ Mix kit (TaKaRa) according to the manual. The primer mixes were loaded in duplicate with SYBR Green PCR Master Mix and $1 \mu \mathrm{g}$ (final) cDNA in 96-well plates. Normalization and fold changes were calculated using the $\Delta \Delta \mathrm{Ct}$ method (Guenou et al. 2009). The primers used are listed in Supplementary Table S1.

\section{Histological and immunochemical analysis}

Cells were fixed in $4 \%$ paraformaldehyde and blocked for $30 \mathrm{~min}$ in $3 \% \mathrm{BSA} / \mathrm{PBS}$. All primary antibodies were diluted in blocking buffer $(0.1 \% \mathrm{BSA} / \mathrm{PBS})$ and incubated with samples overnight at $4{ }^{\circ} \mathrm{C}$. The cells were then incubated with fluorescently labeled secondary antibodies for $1 \mathrm{~h}$. The cell nuclei were counter stained with DAPI (Southern Biotech, Birmingham, USA). All images were captured using a fluorescence microscope (Leica DM 2500). For hematoxylin-eosin staining, the gel was fixed in $4 \%$ paraformaldehyde, dehydrated, and embedded in paraffin.

The primary antibodies used for immunohistochemistry were as follows: anti-EDA (Santa Cruz), antiEDAR (Santa Cruz), anti-K8 (Abcam), and anti-CEA (eBioscience). The secondary antibodies used were anti-mouse-PE (Santa Cruz) and anti-rabbit-PE (Santa Cruz).

Three-dimensional culture of SG cells

Collagen I from rat tails (Gibco) and Matrigel (BD) were used to fabricate successive acellular collagen matrix layers on a polycarbonate membrane. The collagen I was prepared as previously described (Carlson et al. 2008). After the acellular collagen matrix was solidified by placing it at room temperature for approximately $20 \mathrm{~min}$, a cellular collagen matrix layer mixed with fibroblasts and SG cells was added on top of the acellular collagen matrix. The mixture was then incubated for $1 \mathrm{~h}$ at $37^{\circ} \mathrm{C}$. After the mixture was solidified, Epilife medium (Gibco) supplemented with $50 \mathrm{ng} / \mathrm{ml}$ EGF was used to cover the surface of the gel for 21 days, changing the medium every 2 days. After 21 days of culture, the gel was collected for subsequent experiments.
To examine whether Shh influenced the formation of sweat gland tubule-like structures, three groups were designed for comparison: normal 3D culture medium, medium supplemented with $40 \mathrm{ng} / \mathrm{ml}$ Shh recombinant protein (Peprotech), or medium supplemented with Shh antagonist, $20 \mu \mathrm{mol} / \mathrm{L}$ cyclopamine (Sigma). During 3D culture, Shh recombinant protein or Shh antagonist was added to both the gel and the culture medium.

Counting standards for sweat gland-like structures formed by SG cells

To count the number of tubule-like structures formed in the gel, we developed a protocol to quantify these structures. We selected three sections randomly with an interval of at least $100 \mu \mathrm{m}$ (to avoid the same structure being counted repeatedly). The counting methods were as follows: sweat gland-like structures formed by SG cells are defined as a structure composed of 6-15 cells in one region, with a clear lumen that is similar to the transverse section of normal human SG tissue. The number of structures was confirmed under the optical microscope.

Fibroblasts and SG cells labeled with GFP reporter gene and sorted from gel

A lentivirus containing the GFP gene (LV-EGFP) was obtained from Sidansai Stem Cell Technology Co. at a titer of $8 \times 10^{6} \mathrm{IU} / \mathrm{ml}$. We seeded $2 \times 10^{5}$ cells and added $1 \times 10^{6} \mathrm{IU} / \mathrm{ml}$ of LV-EGFP with $10 \mathrm{mg} / \mathrm{ml}$ Polybrene. The medium with LV was removed after $24 \mathrm{~h}$. When the cells were nearly $80 \%$ confluent, they were observed under a fluorescence microscope to confirm the ratio of GFP positive cells.

After cells were cultured in gel for 3 weeks, the gel was digested by collagenase type IV $(2.5 \mathrm{mg} / \mathrm{ml})$ for $1 \mathrm{~h}$ at $37{ }^{\circ} \mathrm{C}$. Then suspension with cells and small pieces gel was stewing for $5 \mathrm{~min}$. Cells in supernatant were collected for sorting. GFP-positive cells were sorted by fluorescence-activated cell sorting (FACS) Calibur (Becton-Dickinson, Franklin Lakes, NJ) for the subsequent experiments.

ELISA analysis and western blot on fibroblasts

Fibroblasts and SG cells co-cultured supernatant was collected for western blot and ELISA analysis. For 
western blot, $5 \mathrm{ml}$ co-cultured supernatant was collected and enriched by centrifuging with 12,000 rpm for $10 \mathrm{~min}$. Anti-Sonic Hedgehog antibody (Abcam) was used to detect Shh in supernatant. For ELISA analysis, co-cultured supernatant were collected at 1 , 2, 3, 4 and 5 day respectively. A Sonic Hedgehog Human ELISA kit (Abcam) was used to detect Shh concentration in supernatant, according to the manual.

\section{Results}

Sweat gland-like structures formed in 3D culture are influenced by human fibroblasts

To find the optimal density of fibroblasts needed for our 3D culture model, we seeded different densities of fibroblasts with $2.5 \times 10^{4} \mathrm{SG}$ cells and counted the number of sweat gland tubule-like structures that formed in the gel. As the results show, seeding $1 \times 10^{5}$ fibroblasts formed the most structures (Fig. 1b). This suggests that $1 \times 10^{5}$ fibroblasts is the optimal density for our 3D culture model.

With the optimal density of fibroblasts, we compared three different SG cell lines, counting the number of structures formed in the 3D culture model. As the results show, tubule-like structures did not form without the presence of fibroblasts, whereas with the presence of fibroblasts, the numbers of sweat gland tubule-like structures formed were 23, 22 and 24 (Fig. 1c). The above results showed that the ability to form structures by different sweat gland cell lines was similar, however fibroblasts were necessary during the process of forming sweat gland tubule-like structures in $3 \mathrm{D}$ culture.

To observe whether human fibroblasts have a role during 3D culture, human fibroblasts and SG cells were seeded within the gel (in which Matrigel and collagen I were mixed in a 1:1 ratio) for 3 weeks. Immunohistochemical analysis showed that with human fibroblasts in the gel, SG cells could form tubule-like structures organized in a manner similar to skin-derived human sweat gland. However, without fibroblasts in the gel, SG cells could not form tubule-like structures (Fig. 1a).

We also used immunofluorescence staining to confirm the structures formed in the gel (Fig. 1d). SG cells express the sweat gland secretory region makers EDA, EDAR, K8 and CEA. Altogether, these results show that with the help of fibroblasts, SG cells have the ability to form mature tubule-like structures.

Shh is secreted by human dermal fibroblasts

During the development of sweat glands, the Shh pathway is important for the formation of the secretory region of the sweat gland (Cui et al. 2014). To find the source of Shh in our 3D culture model, we first performed RT-PCR on primary fibroblasts from dermal. We found that three different lines of fibroblasts all expressed Shh (Fig. 2a). Then we separated fibroblasts which co-cultured with SG cells and cultured those fibroblasts. Immunofluorescence analysis confirmed the presence of Shh protein in fibroblasts (Fig. 2b). To confirm the presence of Shh protein, we collected supernatant form fibroblasts co-cultured with SG cells in vitro for western blot and ELISA analysis. The result demonstrated that Shh was detected in the supernatant (Fig. 2c, d).

To further confirm that fibroblasts secrete Shh in the gel culture, a GFP reporter gene was introduced into fibroblasts using a lentiviral vector to trace the cells in subsequent experiments. Observing the GFP lentivirus-treated fibroblasts (GFP-Fib) under a fluorescence microscope indicated that almost all the cells were labeled with GFP (Fig. 2e). We then used these GFP-Fib cells to do the 3D culture for SG cells. After 3 weeks, GFP-Fib cells were digested from the gel and sorted based on GFP fluorescence. Real time PCR analysis was carried to detect Shh gene expression in fibroblasts, compared with GFP-Fib cells cultured in the gel without SG cells and fibroblasts not cultured in the gel. The results showed that GFP-Fib cells in the gel with SG cells had a higher expression of Shh. Next, we labeled SG cells with GFP-lentivirus (GFP-SG) and observed these cells under a fluorescence microscope to ensure efficient GFP labeling. GFP-SG cells were then seeded in a gel with fibroblasts. After 3 weeks in 3D culture, the GFP-SG cells were digested and sorted for real-time PCR analysis, compared with GFP-SG cells cultured in the gel without fibroblasts and SG cells not cultured in the gel. In the Shh pathway, Smoothened (Smo) is the receptor of Shh, Gli-1 and Gli-2 is downstream Shh pathway genes (Ingham and Placzek 2006). The real time PCR analysis results showed that GFP-SG cells had a higher expression of Smo, Gli-1 and Gli-2. 
$\mathbf{A}$

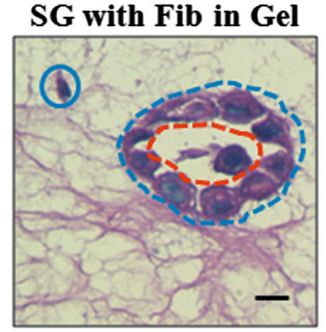

\section{B}

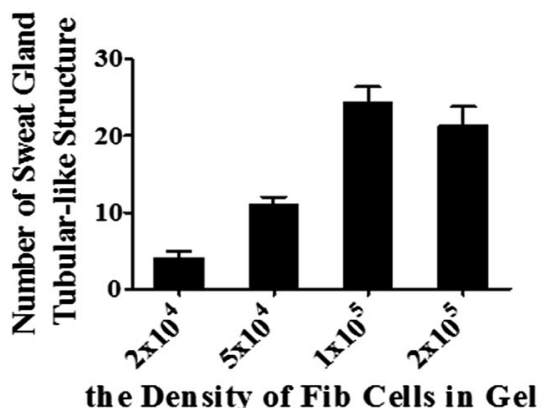

D
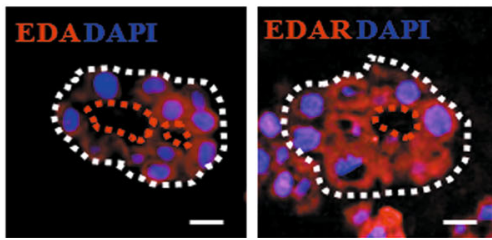

Fig. 1 Sweat gland-like structures formed in 3D culture are influenced by human fibroblast cells. a H\&E images of sweat gland tubule-like structures in gels with or without fibroblasts, and the normal human sweat gland structure. Fibroblasts are indicated with a blue circle, the shape of the tubule-like structure with a blue dashed circle, and the lumen of the tubule-like structure with a red dashed circle. Scale bar $15 \mu \mathrm{m}$. b The number of sweat gland tubule-like structures formed in culture with different densities of fibroblasts with a fixed density of SG cells (see methods for quantification) $(n=3)$. c Comparison of

The above data suggest that Shh is secreted by fibroblasts within the 3D culture. Fibroblasts are likely to interact with SG cells within the gel; fibroblasts secrete Shh, which binds to its receptor Smo on the SG cells, thus activating the Shh pathway, promote the formation of sweat gland tubule-like structures. The SG cells can then, in turn, stimulate the fibroblasts to secrete more Shh and act on SG cells. Those data also suggest that during 3D culture, co-cultured with SG cells was conducive to fibroblasts secrete Shh; and cocultured with fibroblasts, Shh receptor Smo was higher expressed on SG cells.
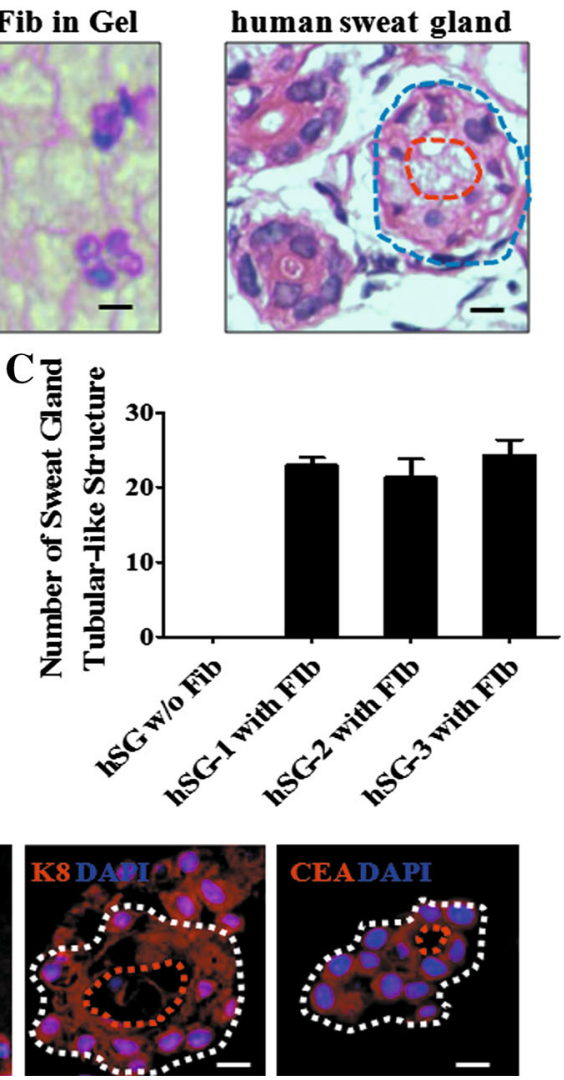

the number of sweat gland tubule-like structures formed by three different fibroblast cell lines with a fixed density of SG cells $(\mathrm{n}=3)$. d Immunofluorescence staining of sweat gland tubulelike structures formed by fibroblasts and sweat gland cells for sweat gland-related makers: EDA, EDAR, K8 and CEA (red); nuclei (blue) were stained with DAPI. The shape of the tubulelike structures is indicated by a white dashed circle and the lumen of the tubule-like structures by a red dashed circle. Scale bar $15 \mu \mathrm{m}$. (Color figure online)

Shh promotes SG cell maturation and enhances the efficiency of structure formation

To examine whether Shh influences the formation of sweat gland tubule-like structures, we compared three experimental groups (normal 3D culture medium, and medium supplemented with either recombinant Shh protein or a Shh antagonist). The concentration of Shh was optimized via concentration titration. We used different concentration of Shh during 3D culture, and counted the numbers of sweat gland tubule-like structure formed. The results indicated that the number 
A

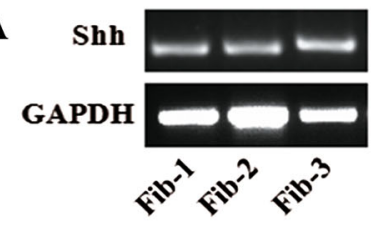

C

Shh

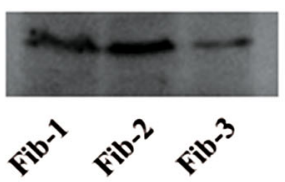

B

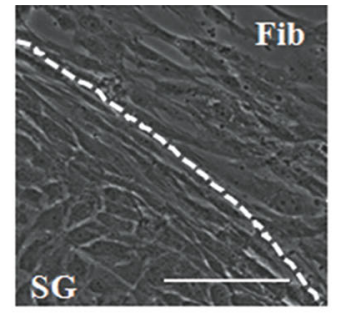

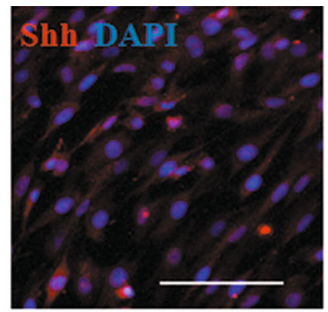

D

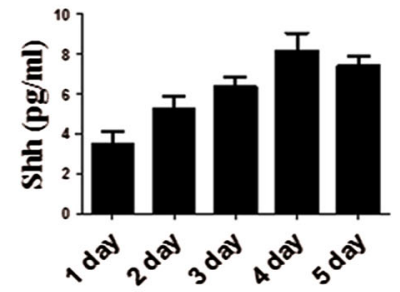

$\mathbf{E}$
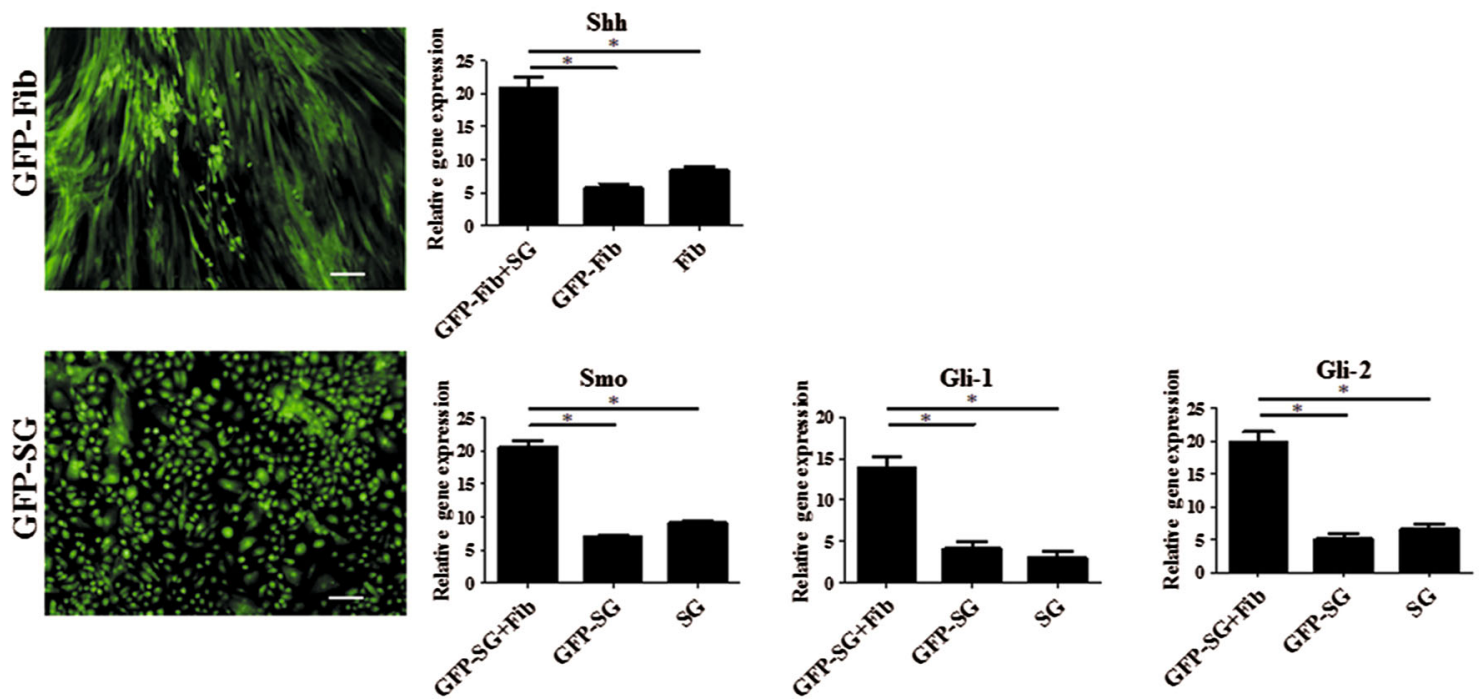

Fig. 2 Shh is secreted by human dermal fibroblasts. a PCR analysis of three different fibroblast cell lines for Shh gene expression. All three lines expressed Shh. b Morphology of cocultured fibroblasts and SG cells. Fibroblasts and SG cells were divided by white dashed circle. Immunofluorescence staining of fibroblasts for Shh (red); nuclei (blue) were stained with DAPI. Scale bar $40 \mu \mathrm{m}$. c Western blot analysis of supernatant form fibroblasts co-cultured with SG cells, the result revealed that all three lines expressed Shh. d ELISA analysis on the collected supernatant also detected the presence of Shh $(n=3)$. e A GFP

of structure was related with concentration of Shh and $40 \mathrm{ng} / \mathrm{ml}$ was the optimal concentration (Fig. 3b).

After 3 weeks of culture, immunohistochemistry analysis was used to confirm the effect of Shh. The results showed that when Shh was added, more tubulelike structures were formed compared with normal 3D culture. Addition of the Shh antagonist, it was hard to find any tubule-like structures (Fig. 3a). The numbers reporter gene was introduced into fibroblasts and sweat gland cells using lentivirus. Cells were observed under a fluorescence microscope, Scale bar $40 \mu \mathrm{m}$. For the fibroblasts, quantitative analysis of Shh mRNA expression (GFP-Fib cells co-cultured with SG cells in the gel, without SG cells in the gel and fibroblast cells). For the SG cells, quantitative analysis of mRNA expression of Smo, Gli-1 and Gli-2 on SG cells (GFP-SG cells co-cultured with fibroblasts, without fibroblasts and SG cells). (Color figure online)

of sweat gland tubule-like structure formed in 3D culture are shown in Fig. 3b. After 3 weeks, To detect gene expression of SG cells in the gel, GFP-SG cells were digested and sorted for real-time PCR analysis. The results showed that, with the addition of Shh, the expression of sweat gland-related genes (K8, CEA, EDA, EDAR) was significantly enhanced. The EDA/ EDAR pathway downstream genes Dkk4 and Wnt10b 
A
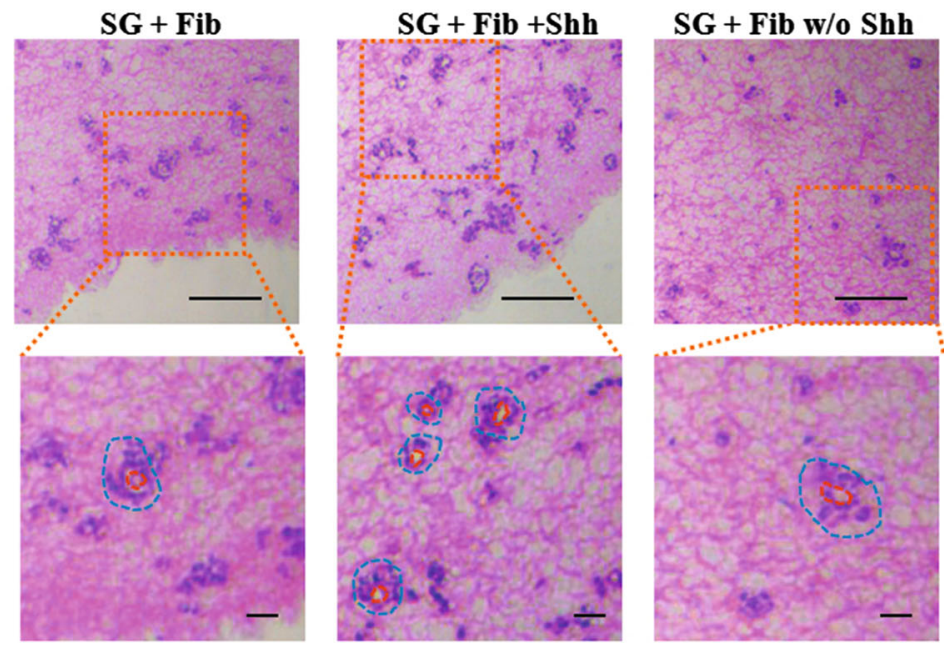

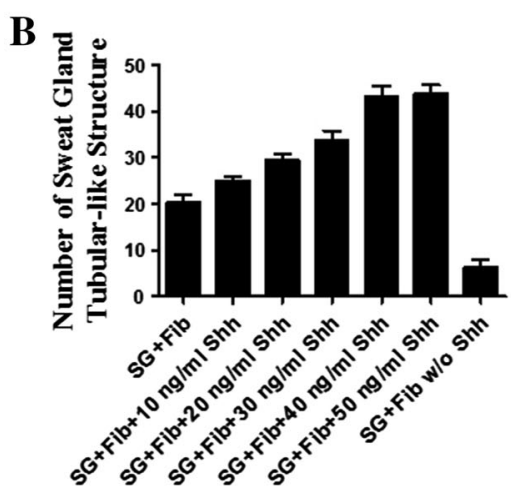

C
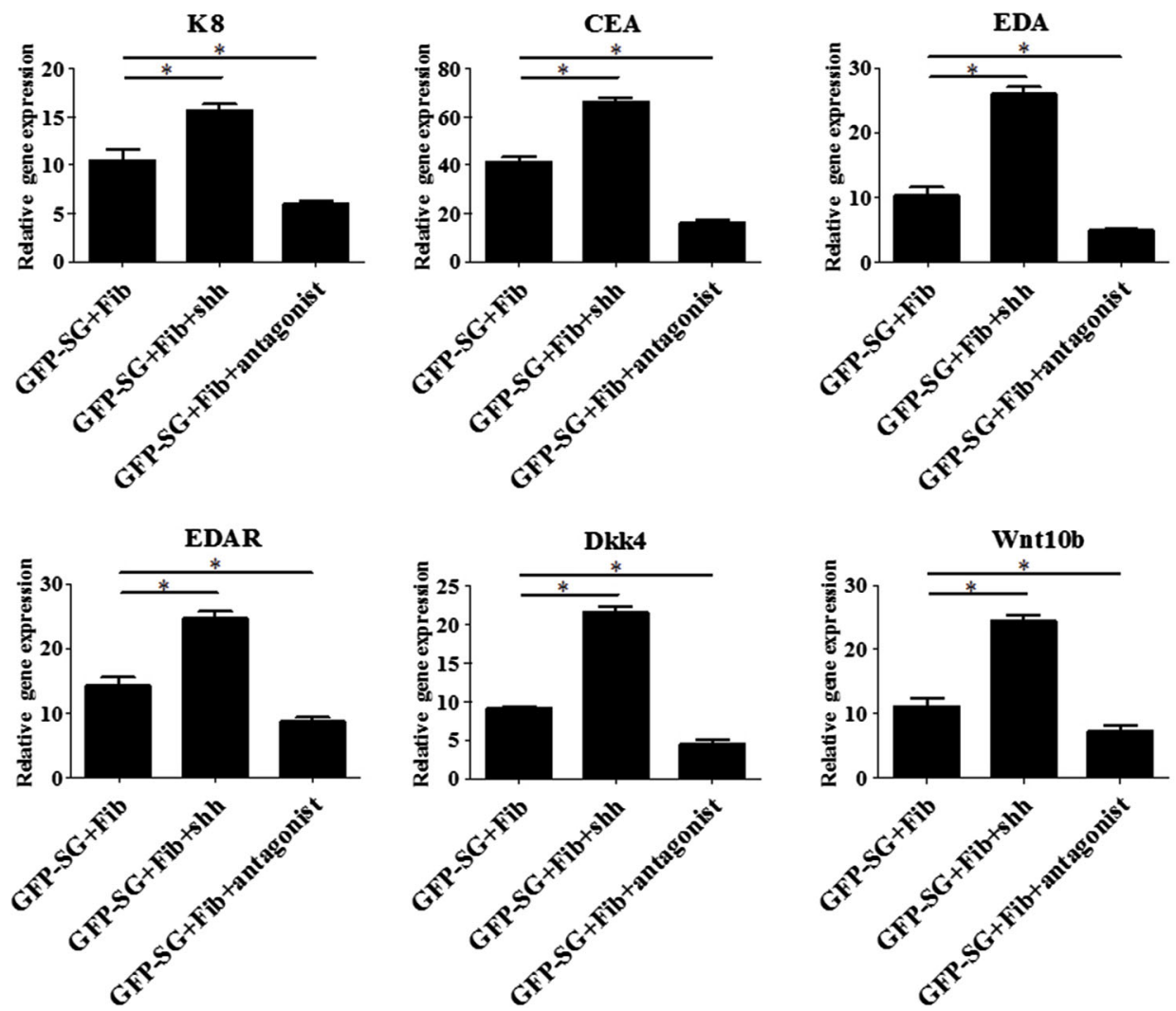

Fig. 3 Shh promotes SG cell maturation and enhances the efficiency of structure formation. a H\&E images of sweat gland tubule-like structures in the gel for three groups (normal 3D culture medium, medium supplemented with Shh protein, or Shh antagonist). The shape and the lumen of the tubule-like structure are indicated by a blue dashed circle and a red dashed circle, respectively. Scale bar $15 \mu \mathrm{m}$. b The number of sweat gland tubule-like structures formed in the three medium conditions (see methods for quantification, $\mathrm{n}=3$ ). The Shh protein added in the medium were 10, 20,30,40, $50 \mathrm{ng} / \mathrm{ml}$ respectively. c Quantitative analysis of mRNA expression of sweat gland development-related makers (EDA, EDAR, K8, CEA) and EDA/EDAR pathway downstream gene (Dkk4, Wnt10b) of the three different groups $(n=3)$. (Color figure online) 
also had a higher expression, whereas with the addition of a Shh antagonist, the expression of these genes was reduced (Fig. 3c). The above data suggest that Shh is an important factor during the formation of sweat gland tubule-like structures in 3D culture; it can promote SG cell maturation and can enhance the efficiency of structure formation by adding extra Shh recombinant protein.

\section{Discussion}

In this study, we used a 3D culture model in vitro to confirm the effect of fibroblasts on the formation of sweat gland tubule-like structures. With the help of fibroblasts, sweat gland cells could form tubule-like structures. We demonstrated that fibroblasts secrete Shh in the 3D culture and that fibroblasts interact with SG cells when co-cultured in the gel. We also found that Shh was an important factor during the process of structure formation, promoting SG cells maturation and adding extra Shh can enhance the efficiency of structure formation.

Fibroblasts have an interesting relationship with cells in the skin. In the study of epidermis reconstitution, the number of fibroblasts within the collagen matrix was a critical factor for the establishment of the epidermis (Ghalbzouri et al. 2002), and fibroblasts assisted in the reconstitution of a stratified epidermis (Biedermann et al. 2010). Fibroblasts were also shown to have an important role in wound healing (Bartold and Raben 1996; Werner et al. 2007; Spiekstra et al. 2007). During the development of sweat gland, in the first place a cellular bud of gland grow into the mesenchyme form the duct of sweat gland, then the duct continue down grow into the mesenchyme and the terminal part coil form the secretory part of sweat gland (Cui et al. 2014). Before the duct and the secretory part form, fibroblasts are already existed in dermal, and provide the environment which is needed by duct and secretory part formation. Our research demonstrated that fibroblast was a necessary factor during the process of forming sweat gland tubule-like structures in 3D culture. This was similar to the previous research that fibroblast played an important role in development of skin.

Our studies also find that fibroblasts secrete Shh in the $3 \mathrm{D}$ culture and promote sweat gland cells form tubule-like structures, and fibroblasts have interacted with SG cells During sweat gland development, it has been demonstrated that EDA/EDAR signaling is important during the development of sweat gland (Mustonen et al. 2003; Tucker et al. 2000; Cui et al. 2009). Shh is downstream of EDA/EDAR and a key to the development of several skin appendages (Chuong et al. 2000). In hair follicles, Shh is essential for the normal advancement beyond the hair germ stage of development (Chiang et al. 1999); in salivary glands, Shh is required for the late stage of branching morphogenesis (Jaskoll et al. 2004); and in sweat glands, Shh is required for the final formation of the secretory region (Cui et al. 2014). These previous findings are consistent with our study that Shh can promote sweat gland cells form tubule-like structures in the $3 \mathrm{D}$ culture and enhance the efficiency of sweat gland tubule-like structure formation.

Tissue engineering has developed rapidly in recent years, providing many benefits for patients (Griffith and Naughton 2002; Böttcher-Haberzeth et al. 2010; Priya et al. 2008; Groeber et al. 2011). Future developments in skin tissue engineering should include the addition of skin appendages. Based on our research, we can enhance the efficiency of sweat gland formation in 3D culture; we can optimize the identification of functional sweat gland; we also provide a model for drug test on sweat gland in vitro.

Acknowledgments Funding for this research was provided by The Project of Soochow Science and Technology Plan (SYS201437). The Natural Science Foundation of Jiangsu Province (BK20131156), The Jiangsu Provincial Special Program of Medical Science (BL2013015).

Open Access This article is distributed under the terms of the Creative Commons Attribution 4.0 International License (http:// creativecommons.org/licenses/by/4.0/), which permits unrestricted use, distribution, and reproduction in any medium, provided you give appropriate credit to the original author(s) and the source, provide a link to the Creative Commons license, and indicate if changes were made.

\section{References}

Bartold PM, Raben A (1996) Growth factor modulation of fibroblasts in simulated wound healing. J Periodontal Res 31:205-216

Biedermann T, Pontiggia L, Böttcher-Haberzeth S et al (2010) Human eccrine sweat gland cells can reconstitute a stratified epidermis. J Invest Dermatol 130:1996-2009 
Böttcher-Haberzeth S, Biedermann T, Reichmann E (2010) Tissue engineering of skin. Burns 36:450-460

Burd A, Chiu T (2005) Allogenic skin in the treatment of burns. Clin Dermatol 23:376-387

Carlson MW, Alt-Holland A, Egles C, et al (2008) Three-dimensional tissue models of normal and diseased skin. Curr Protoc Cell Biol, Chapter 19: Unit 19.9

Chiang C, Swan RZ, Grachtchouk M et al (1999) Essential role for Sonic hedgehog during hair follicle morphogenesis. Dev Biol 205:1-9

Chuong CM, Patel N, Lin J et al (2000) Sonic hedgehog signaling pathway in vertebrate epithelial appendage morphogenesis: perspectives in development and evolution. Cell Mol Life Sci 57:1672-1681

Cui Chang-Yi, Kunisada Makoto, Esibizione Diana et al (2009) Analysis of the temporal requirement for Eda in hair and sweat gland development. J Invest Dermatol 129:984-993

Cui CY, Yin M, Sima J et al (2014) Involvement of Wnt, Eda and Shh at defined stages of sweat gland development. Development 141:3752-3760

Cuono C, Langdon R, McGuire J (1986) Use of cultured epidermal autografts and dermal allografts as skin replacement after burn injury. Lancet 17:1123-1124

Fu X, Li X, Cheng B, Chen W, Sheng Z (2005) Engineered growth factors and cutaneous wound healing: success and possible questions in the past 10 years. Wound Repair Regen 13:122-130

Fu XB, Fang LJ, Li XK, Cheng B, Sheng Z (2006) Enhanced wound-healing quality with bone marrow mesenchymal stem cells autografting after skin injury. Wound Repair Regen 14:325-335

Ghalbzouri A, Gibbs S, Lamme E, Van Blitterswijk CA, Ponec M (2002) Effect of fibroblasts on epidermal regeneration. Br J Dermatol 147:230-243

Griffith LG, Naughton G (2002) Tissue engineering-current challenges and expanding opportunities. Science 295: 1009-1014

Groeber F, Holeiter M, Hampel M, Hinderer S, Schenke-Layland K (2011) Skin tissue engineering - in vivo and in vitro applications. Adv Drug Deliv Rev 63:352-366

Guenou H, Nissan X, Larcher F et al (2009) Human embryonic stem cells derivatives enable full reconstruction of the pluristratified epidermis. Lancet 374:1745-1753

Huang Sha, Yongan Xu, Changhao Wu, Sha Deqian, Xiaobing $\mathrm{Fu}$ (2010) In vitro constitution and in vivo implantation of engineered skin constructs with sweat glands. Biomaterials 31:5520-5525

Ingham PW, Placzek M (2006) Orchestrating ontogenesis, variations on a theme by sonic hedgehog. Nat Rev Genet $7: 841-850$

Jaskoll T, Leo T, Witcher D et al (2004) Sonic hedgehog signaling plays an essential role during embryonic salivary gland epithelial branching morphogenesis. Dev Dyn 229:722-732

Kleinman HK, Martin GR (2005) Matrigel: basement membrane matrix with biological activity. Semin Cancer Biol 15:378-386

Li H, Chen L, Zhang M, Tang S, Fu X (2013) Three-dimensional culture and identification of human eccrine sweat glands in matrigel basement membrane matrix. Cell Tissue Res 354:897-902

Mustonen T, Pispa J, Mikkola ML et al (2003) Stimulation of ectodermal organ development by Ectodysplasin-A1. Dev Biol 259:123

Naoum JJ, Roehl KR, Wolf SE, Herndon DN (2004) The use of homograft compared to topical antimicrobial therapy in the treatment of second-degree burns of more than $40 \%$ total body surface area. Burns 30:548-551

Priya SG, Jungvid H, Kumar A (2008) Skin tissue engineering for tissue repair and regeneration. Tissue Eng Part B Rev 14:105-118

Saga K (2002) Structure and function of human sweat gland studied with histochemistry an cytochemistry. Prog Histochem Cytochem 37:323-386

Sheng Z, Fu X, Cai S, Lei Y, Sun T, Bai X, Chen M (2009) Regeneration of functional sweat gland-like structures by transplanted differentiated bone marrow mesenchymal stem cells. Wound Repair Regen 17:427-435

Spiekstra SW, Breetveld M, Rustemeyer T, Scheper RJ, Gibbs S (2007) Wound-healing factors secreted by epidermal keratinocytes and dermal fibroblasts in skin substitutes. Wound Repair Regen 15:708-717

Tucker AS, Headon DJ, Schneider P et al (2000) Edar/Eda interactions regulate enamel knot formation in tooth morphogenesis. Development 127:4691-4700

Werner S, Krieg T, Smola H (2007) Keratinocyte-fibroblast interactions in wound healing. J Invest Dermatol 127:9981008 